\title{
MR Imaging Spectrum of Neuro-rhino-sino-orbital Fungal Infections in Patients Treated for COVID
}

\author{
Sushil Kachewar $P \boldsymbol{h D}^{1}$ iD, Smita Kachewar $M D^{2}$ iD
}

${ }^{1}$ Department of Radio-diagnosis, Dr Vikhe Patil Foundation's Medical College, Vildghat, Ahmednagar, Maharashtra, India ${ }^{2}$ Department of Pathology, Deenanath Mangeshkar Hospital, Erandwane, Pune, Maharashtra, India

Date of submission: $5^{\text {th }}$ June 2021

Date of acceptance: $31^{\text {st }}$ October 2021

Date of publication: $1^{\text {st }}$ December 2021

\section{Abstract}

Introduction: Doctors across the globe need to stay abreast with daily changing complications occurring in patients already infected with COVID. Mucormycosis is one such infection affecting COVID patients and creating havoc. If detected early, dreaded complications can be prevented. Hence this study was undertaken to present and analyse the spectrum of imaging findings of fungal infection as seen in MRI images of patients who were previously treated for COVID.

Methods and Materials: The different patterns of findings of Neuro-rhino-sino-orbital fungal infections as seen in MRI images, in 100 patients, who received standard treatment for COVID in the recent past and presented with suggestive signs and symptoms. This study proposes a new staging system [Stage $0, \mathrm{X}, \mathrm{I}, \mathrm{II}, \mathrm{III}, \mathrm{IV}]$ for describing the affected region by fungal infection.

Results: No positive MRI findings to suggest any neural tissue, orbital or sino-nasal involvement was seen in 35 $\%$ of cases. Only sino-nasal involvement was noted in $23 \%$ of cases. Sino-nasal as well as orbital involvement was seen in $21 \%$ of cases. Neuro-rhino-sino-orbital lesions were seen in $11 \%$ cases. Non fungal orbito-facial soft tissue inflammation was seen in $10 \%$ of cases.

Conclusion: In patients who have received standard treatment for COVID and who later developed signs and symptoms of neural, orbital or sino-nasal involvement; positive imaging findings were noted in $65 \%$ cases. Fungal infections were seen more in middle aged population [40-60 years of age]. About $75 \%$ of affected patients were known diabetics. Neural involvement was least common.

Key words: Coronavirus disease, COVID- 19, Diabetes, Fungal infection, MRI, Mucormycosis, Neuroimaging, Orbital infection, Pandemic, Sino nasal infection.

\section{Introduction}

Tncreasing cases of fungal infections like mucormycosis are being reported in patients who were treated for

Access this article online
Website: https://www.nepjol.info/index.php/NJN
DOI: https://doi.org/10.3126/njn.v18i4.37486
HOW TO CITE
Kachewar S, Kachewar S. MR Imaging Spectrum of Neuro-rhino-
sino-orbital Fungal Infections in Patients Treated for COVID.
NJNS. 2021;18(4):19-25.

Address for correspondence:

Dr. Sushil Kachewar

Department of Radio-diagnosis,

Dr Vikhe Patil Foundation's Medical College,

Vi-ladghat, Ahmednagar, Maharashtra, India.

E-mail: sushilkachewar@hotmail.com

Phone: +91-9921160357

Copyright (C) 2021 Nepalese Society of Neurosurgeons (NESON)

ISSN: 1813-1948 (Print), 1813-1956 (Online)

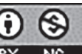

This work is licensed under a Creative Commons Attribution-Non Commercial 4.0 International License.
COVID-19 infection due to resulting immunosuppression and various comorbidities like diabetes, that increase the vulnerability of such patients to secondary infections. ${ }^{1}$ In a retrospective study of SARS and influenza data from China and worldwide, it was noted that not only mucormycosis but aspergillosis, candidiasis or cryptococcosis may individually or in various combinations may affect such patients. Hence, active search by microbiologists and pathologists is mandatory, else they might be missed or misdiagnosed. ${ }^{2}$

By May 28, 2021, a total of 168,599,045 confirmed COVID-19 cases, including 3,507,477 deaths have been reported worldwide to the World Health Organization (WHO). ${ }^{3}$ Indian contribution to this global statistics has been 27,555,457 confirmed cases and about 318,895 deaths. ${ }^{4}$ However, no data pertaining to occurrence of fungal infections in patients who have been treated for COVID is available as of now.

Inhalation of sporangiospores, or consumption of contaminated food or traumatic inoculation are the routes by which fungi infect human beings. ${ }^{5,6}$ Angio-invasion, therefore mortality makes mucormycosis a deadly disease. ${ }^{7.8}$ Traditionally these fungal infections have been increasingly reported in cases of diabetes mellitus, 
haematological malignancies, solid organ transplants, and corticosteroid therapy. ${ }^{8,9,10,11,12}$

Interestingly, prior to COVID there existed a difference in risk factors between the western world and Asian countries. ${ }^{7}$ While haematological malignancies and organ transplants accounted for most cases in Europe and USA, diabetes was dominant in Asian cases. ${ }^{13,14,15}$ At present, COVID infection appears to be the prevalent risk factor.

Tremendous diagnostic data is available for diagnosing COVID-19 pneumonia, and numerous guidelines are being put forth for timely diagnosis and prompt treatment. But diagnostic data and different imaging appearances about patients treated for COVID and suffering from fungal infections are not available. Hence, this retrospective study was done to analyse the spectrum of neuro-rhinosino-orbital fungal Infections as seen in MRI images of patients treated for COVID.

\section{Methods and Materials}

This retrospective observational study was approved by the institutional ethical committee.

Patients who had been treated for COVID-19 and developed sino-naso-orbital region related symptoms within six months from January to May, 2021, were included using the following criteria:

a. Positive RT-PCR for SARS- CoV-2 obtained with nasopharyngeal / oropharyngeal swabs or COVID Positive on RAPID Antigen Test before starting treatment for COVID.

b. Received Steroids during treatment for COVID.

c. New onset sino-naso-orbital region related symptoms not there prior to being affected by COVID.

d. MRI scan done.

e. Histopathological and Microbiological studies to confirm the causative fungal etiology by using $\mathrm{KOH}$ preparation and Periodic Acid Schiff stain.

A total of 100 patients were included in the study between 20-70 years of age.

All patients were imaged using a multi-channel 1.5 T GE MRI machine. Standard sequences for MRI Head, PNS and Orbits included multiplanar T1W, T2W, STIR and post contrast $\mathrm{T} 1 \mathrm{~W}$ in most cases.

The clinical, demographic and imaging data of all the patients were recorded and various parameters were compared.

\section{MRI image analysis}

Pure soft tissue inflammation like Cellulitis and Sinusitis appear uniformly hypointense on $\mathrm{T} 1 \mathrm{~W}$ and hyperintense on $\mathrm{T} 2 \mathrm{~W}$ images. It shows moderate uniform enhancement on post contrast images. But in case of fungal infection, there is focal or diffuse $\mathrm{T} 1 \mathrm{~W}$ iso to hyperintense areas which are iso to hypointense foci on $\mathrm{T} 2 \mathrm{~W}$ and do not show any post contrast enhancement due to presence of fungal hyphae and necrotic areas.

\section{Statistical analysis}

Numerical data were expressed as a percentage (\%) of the total. Relevant data was expressed in tabular form.

\section{Results}

Demographic and clinical characteristics: These are listed in Table 1.

The mean interval between the onset of initial symptoms and MRI was 5-10 days. About $78 \%$ of affected patients were known diabetics. Fungal infections were seen more in the middle aged population [40-60 years of age].

No positive MRI findings to suggest any neural tissue, orbital or sino-nasal involvement was seen in 35 $\%$ of cases. Only sino-nasal fungal involvement was noted in $23 \%$ of cases. Sino-nasal as well as orbital fungal involvement is seen in $21 \%$ of cases. Neuro-sino-nasoorbital fungal lesions were seen in $11 \%$ cases. Non fungal orbito-facial soft tissue inflammation was seen in $10 \%$ cases.

In patients who have received standard treatment for COVID and who later developed signs and symptoms of neural, orbital or sino-nasal involvement; positive imaging findings were noted in $65 \%$ cases. Neural involvement was least common.

Figures 1-4 depict the various MRI findings that we came across from the patients in the current study.

\begin{tabular}{|l|c|c|c|}
\hline \multicolumn{1}{|c|}{ Criteria } & Male & Female & Total \\
\hline $\begin{array}{l}\text { Gender } \\
\text { Symptoms }\end{array}$ & 54 & 46 & 100 \\
\hline $\begin{array}{l}\text { Fever } \\
\text { Facial Pain }\end{array}$ & 17 & 13 & 030 \\
\hline Facial Swelling & 50 & 43 & 093 \\
\hline Nasal Blockage & 38 & 31 & 092 \\
\hline Comorbidities & & & 074 \\
\hline Diabetes & 43 & 35 & 078 \\
\hline Hypertension & 06 & 11 & 017 \\
\hline Kidney Disease & 05 & 02 & 007 \\
\hline Liver Disease & 03 & 01 & 004 \\
\hline Known Malignancy & 03 & 06 & 010 \\
\hline
\end{tabular}

Table 1: Demographic, clinical profile of the patients. 
MRI in Fungal Infections of COVID Cases

\begin{tabular}{l|c|}
\hline \multicolumn{1}{|c|}{ Proposed Staging } & $\%$ cases \\
\hline Stage 0: No Abnormality on MRI & 35 \\
\hline Stage X: Only Orbito-facial Soft Tissue Inflammation & 10 \\
\hline Stage 1: Only Sino-nasal fungal Involvement & 23 \\
\hline Stage 2: Only Sino-naso-orbital Involvement & 21 \\
\hline Stage 3: Neuro Sino-naso-orbital Involvement & 11 \\
\hline Stage 4: Associated CNS Complications & 07 \\
\hline
\end{tabular}

Table 2: Proposed MRI Staging and details of patients
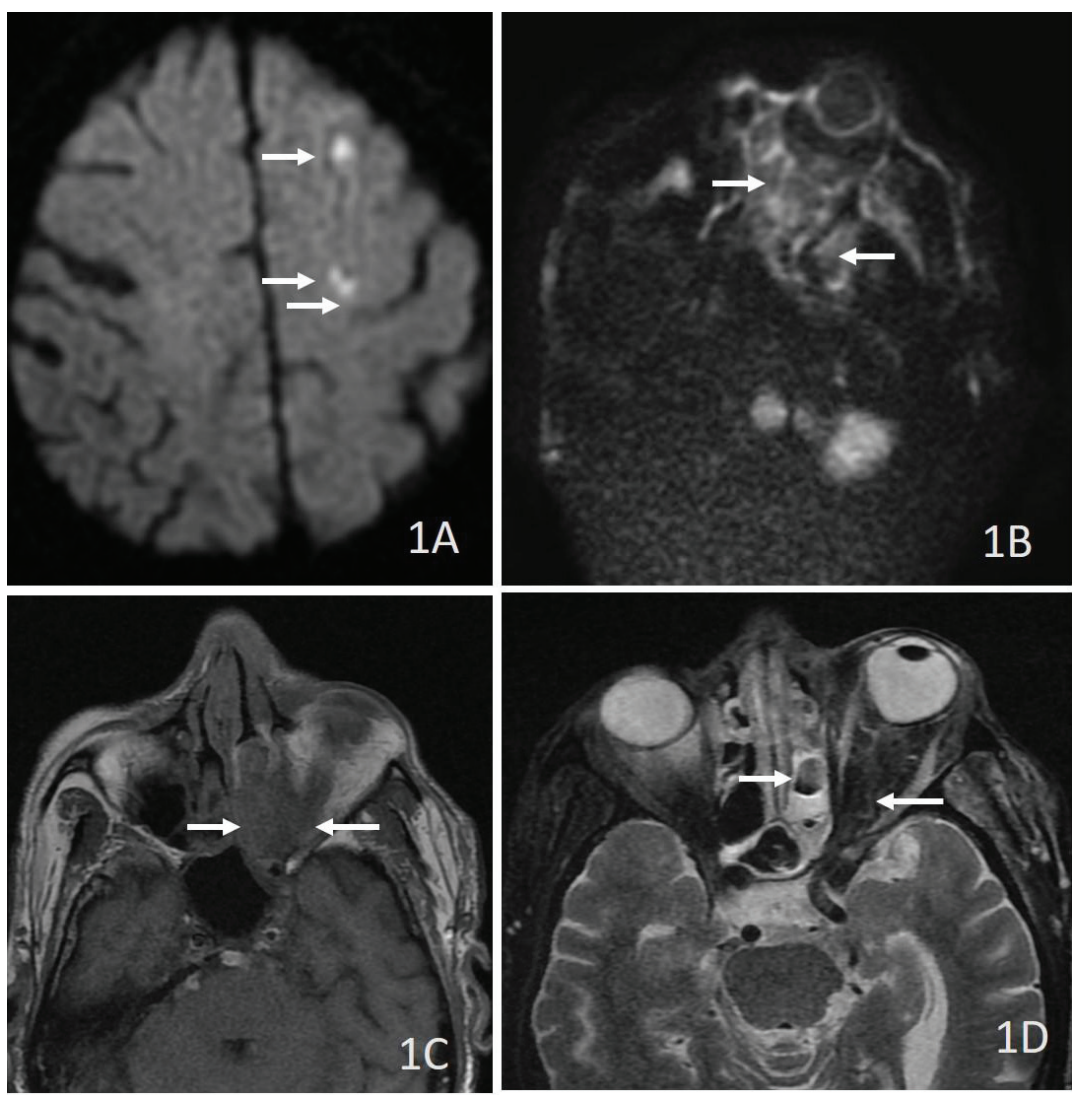

Figure 1: (A) Axial MRI DW image: Acute lacunar infarcts seen as small areas of restricted diffusion in left high frontal region. (B) Axial MRI DWI image: Left intraconal retro orbital soft tissue inflammation. (C) Axial MRI T1W image: Iso to hypo intense fungal soft tissue lesions in left intraconal and retro-orbital region, left middle and posterior ethmoid air cells and at left orbital apex. (D) Axial MRI T2W image: Inflamed mucosa seen as hyperintensity, fungal affected areas stay hypointense as seen in left retro-orbital and left middle ethmoid air cells region. 


\section{Kachewar et al}
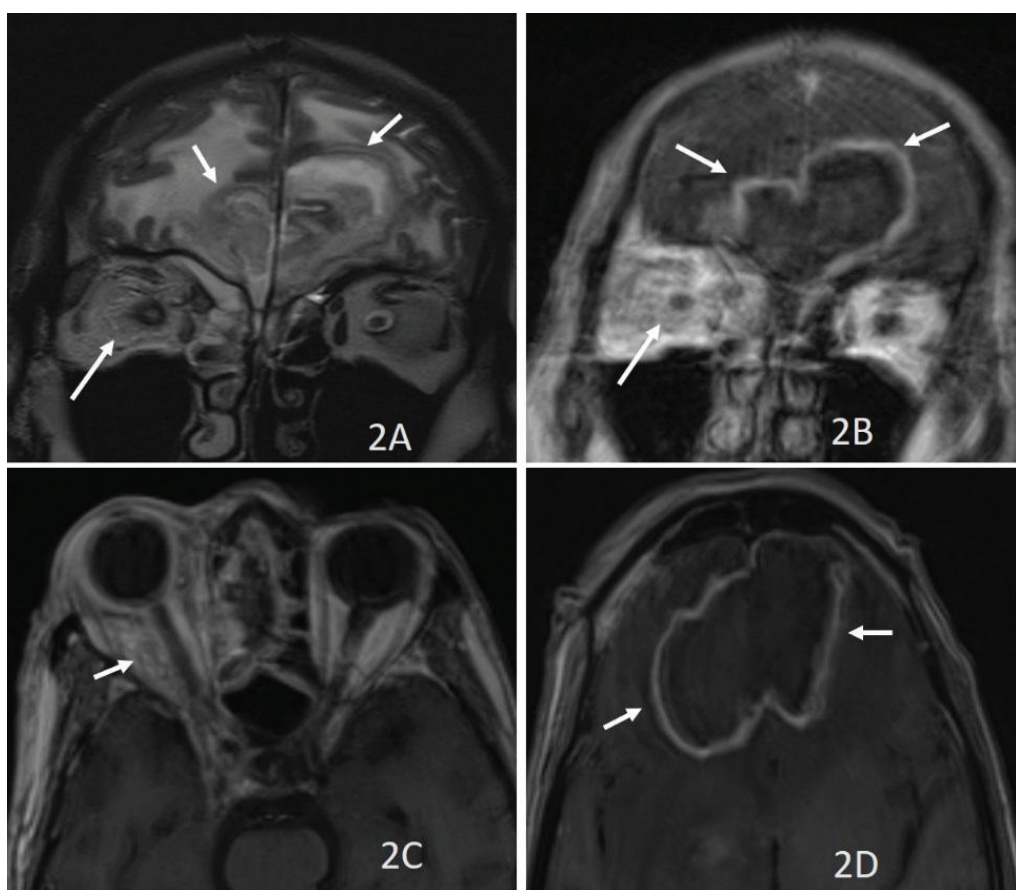

Figure 2: (A) Coronal MRI T2W image: Altered signal intensity areas in frontal region bilaterally and in right periorbital soft tissues. (B) Coronal MRI Post Contrast image: Avidly enhancing thin walls around lesions in frontal region bilaterally [fungal abscesses] and in moderately enhancing right periorbital fungal inflammation. (C) Axial T1W $+C$ image: Right periorbital and retro-orbital hyperintense signal suggesting diffuse fungal infection. (D) Axial MRI Post Contrast image: Avidly enhancing thin walls around lesions in frontal regions bilaterally [fungal abscesses] surrounding the non-enhancing central areas.

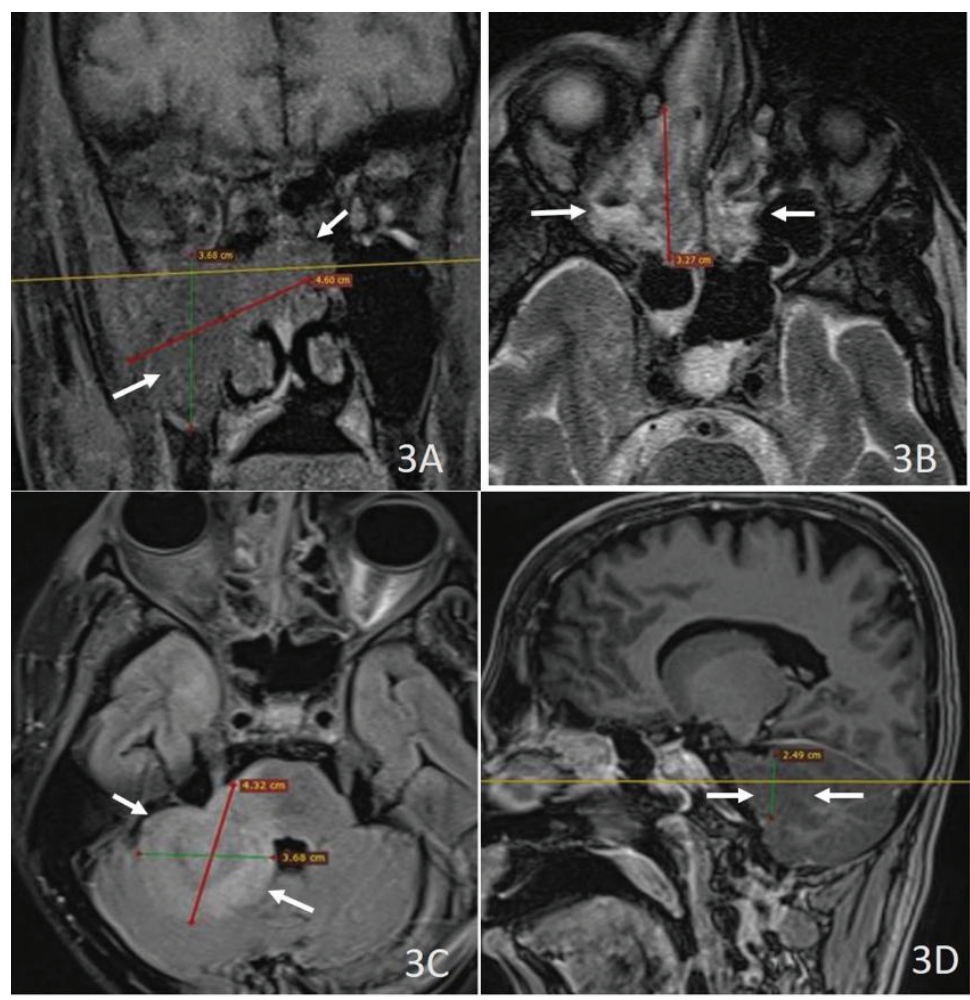

Figure 3: (A) Coronal MRI T1W image: Iso to hypointense abnormal soft tissues invading right maxillary sinus and right ethmoid and nasal cavity suggesting fungal sinusitis. (B) Axial MRI T2W image: Iso to hypointense abnormal soft tissues invading bilateral ethmoid and nasal cavities suggesting fungal Sinusitis. (C) Axial MRI FLAIR image: Isointense fungal mass in right CP angle region. (D) Sagittal MRI T1W image: Isointense fungal mass involving the right lateral pons, right $M C P$ and right cerebellar hemisphere. 

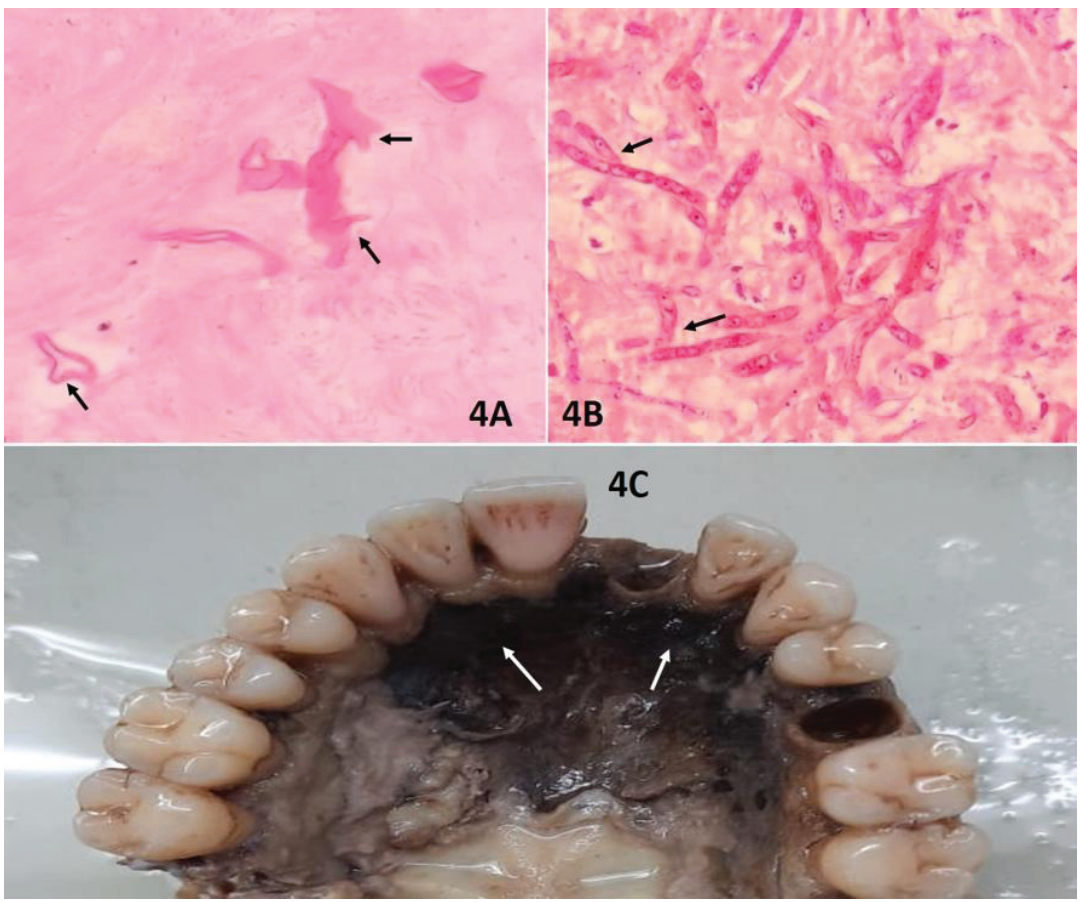

Figure 4: (A) H\& E stain: $40 x$ : Mucormycosis Hyphae: Broad, aseptate showing branching at right angle. (B) H\& E stain: 40 x: Aspergillosis Hyphae: Narrow, septate showing branching at acute angle. (C) Gross specimen of maxilla affected with fungal infection.

\section{Discussion}

Prior to COVID, a global review of mucormycosis cases from January 2000 to 2017 , quoted an incidence of $34 \%$ in Europe, $31 \%$ in Asia and $28 \%$ in North and South America, while Africa (3\%) and Australia and New Zealand (3\%) contributed the least. ${ }^{7}$ But recent studies are pointing to increasing prevalence in Asian countries like India. ${ }^{8,13}$

Mucormycosis is an aggressive opportunistic infection occurring predominantly in immune-compromised patients. ${ }^{16}$ The pathogen is naturally occurring all around us in nature and environment, spread over the body surface and orifices. At first the paranasal sinuses and the nasopharynx of immunocompromised individuals get inoculated by spores which then subsequently spread to the orbit and intracranial cavity by invading the vascular lamina and thereby increasing the inflammation and causing infarction and resultant necrosis. Timely diagnosis and appropriate treatment alone can save both the life as well as the sight. ${ }^{16}$

Present study consisted of 100 patients between 20-70 years of age. Males [54\%] were more commonly affected than females $[46 \%]$. Facial pain and swelling were the most common presenting symptoms [ $>90 \%$ ]. Diabetes was the most common risk factor [78 \%]. Middle aged population was most commonly affected [40-60 years of age].
Although mucormycosis can affect any region in the body, it is broadly classified into rhino-orbito-cerebral (ROCM) form, pulmonary form, gastrointestinal form, cutaneous form, renal form and a disseminated form. Additionally, there are other miscellaneous forms, that include infections of bones, heart, ear, parotid gland, uterus, urinary bladder and lymph nodes too. ${ }^{7}$ In the present study only the cases affecting head, neck and face that are presenting more in current pandemic are included.

No positive MRI findings to suggest any neural tissue, orbital or Sino-nasal involvement was seen in $35 \%$ of cases. In patients who have received standard treatment for COVID and who later developed signs and symptoms of neural, orbital or Sino-nasal involvement; positive imaging findings were noted in $65 \%$ cases.

Local neural involvement was seen in $11 \%$ cases, while distal CNS complications like Infarct / Bleed / CNS fungal mass formation were seen in $7 \%$ cases.

The demographic characteristics of the patients seen in the current study were similar to those reported in studies reported by other researchers. ${ }^{6-10}$ The male gender was more affected than females like other studies. Diabetes was the most common risk factor and all cases were administered steroids for COVID infection. This correlates well with findings reported in other studies of the COVID-19 era. ${ }^{1}$

As of now standard treatment guidelines are being laid for treating such cases. Liposomal amphotericin B 
has been recommended as the first-line therapy for the treatment of mucormycosis along with surgery whenever possible. ${ }^{17}$

It has been found that although debridement of sinuses is necessary in all cases of rhino-orbital cerebral mucormycosis; orbit retention should be attempted in patients with controlled diabetes and non-progressive orbital involvement. ${ }^{18}$

The present study highlights the importance of a high index of suspicion in all follow up cases of COVID, so that Neuro-rhino-sino-orbital infection is diagnosed timely and treated appropriately. Exact amount and duration of steroid treatment during COVID infection could not be ascertained in each case.

Radiological and Histopathological correlation are the strengths of this study. This study proposes a new staging system [Stage 0, X, I, II, III, IV] for describing the affected region by fungal infection which might be of use to operating surgeons and treating physicians and can also help the common man understand the severity of involvement.

Histopathology of the excised or debrided specimens had two main characteristics: One was that -they showed a clear-cut line of demarcation between the infected and non-infected tissues. There was necrosis in the region and thrombosis of micro vessels. Hence studies are needed specifically to ascertain whether the tissue was first dead due to vascular insult and then this dead tissue was invaded by the ubiquitous fungus. Answer to this question would radically change our approach to treatment if this is true, our main focus will be on preventing vaso-occlusion rather than on antifungals. Second characteristic was that extent of fungal involvement was beyond that seen with naked eye or mentioned in imaging reports thereby indicating a high probability of angioinvasive spread rather than contiguous spread.

Limitations of the study are small sample size. Large sample size would be more informative. Also, this study is based from one small geographic location. Multicentric studies from across the globe would add completeness to the scientific spectrum on this entity.

As new cases continue to flood us, in this scenario one thing is clear that to treat such cases it is the teamwork of different clinical specialities like Medicine, Otorhinolaryngology, Ophthalmology, Neurosurgery, Radiology, Pathology and Microbiology amongst others that is most important. ${ }^{19}$ It has been reported that a small delay of even 6 days in initiating treatment shockingly doubles the 30-day mortality from $35 \%$ to $66 \%{ }^{1}$ Immediate treatment with proper consensus and coordination amongst these various specialities alone is mankind's only hope against the phoenix-like emergence of this fungus in a land that is currently invaded by virus.

\section{Conclusion}

Newer data about rapidly evolving fungal infections in patients that received treatment for COVID in the recent past is strongly emerging day in and day out. Hence, wide spread awareness about this entity amongst medical fraternity and general masses is of paramount importance for timely diagnosis and management.

\section{Conflict of Interest: None \\ Source(s) of support: None}

\section{References}

1. Sen M, Lahane S, Lahane TP, Parekh R, Honavar SG. Mucor in a Viral Land: A Tale of Two Pathogens. Indian J Ophthalmol. 2021; 69(2):244-252. https:// doi.org/10.4103/ijo.IJO 377420

2. Song G, Liang G, Liu W. Fungal Co-infections Associated with Global COVID-19 Pandemic: A Clinical and Diagnostic Perspective from China. Mycopathologia. 2020 Aug;185(4):599-606. https:// doi.org/10.1007/s11046-020-00462-9

3. WHO Coronavirus Disease (COVID-19) Dashboard | WHO Coronavirus Disease (COVID-19) Dashboard [Internet]. [cited 2021 May 29]. Available from: https://covid19.who.int

4. COVID-19 Tracker Updates For India For State Wise \&amp; District Wise Data [Internet]. [cited 2021 May 29]. Available from: https://covidindia.org.

5. Ribes JA, Vanover-Sams CL, Baker DJ. Zygomycetes in Human Disease. Clin. Microbiol. Rev. 2000;13:236301. https://doi.org/10.1128/CMR.13.2.236

6. Richardson M. The ecology of the Zygomycetes and its impact on environmental exposure. Clin. Microbiol. Infect. 2009; 15:2-9. https://doi.org/10.1111/j.14690691.2009.02972.x

7. Jeong W, Keighley C, Wolfe R, Lee WL, Slavin MA, Kong DCM, Chen SCA. The epidemiology and clinical manifestations of mucormycosis: A systematic review and meta-analysis of case reports. Clin. Microbiol. Infect. 2019; 25:26-34. http://doi.org/10.1016/j. cmi.2018.07.011

8. Prakash H, Ghosh AK, Rudramurthy SM, Singh P, Xess I, Savio J, Pamidimukkala U et al. A prospective multicenter study on mucormycosis in India: Epidemiology, diagnosis, and treatment. Med. Mycol. 2019;57(4),395-402. http://doi.org/10.1093/mmy/ myy060

9. Rees JR, Pinner RW, Hajjeh RA, Brandt ME, Reingold AL. The epidemiological features of invasive mycotic infections in the San Francisco Bay area, 1992-1993: Results of population-based laboratory active surveillance. Clin. Infect. Dis. 1998; 27:11381147. http://doi.org/0.1093/clinids/27.5.1138 
10. Torres-Narbona M, Guinea J, Martínez-Alarcón J, Muñoz P, Gadea I, Bouza E. Impact of zygomycosis on microbiology workload: A survey study in Spain. $J$. Clin. Microbiol. 2007; 45:2051-2053. https://doi. org/10.1093/clinids/27.5.1138

11. Bitar D, Van Cauteren D, Lanternier F, Dannaoui E, Che D, Dromer F, Desenclos JC, Lortholary O. Increasing incidence of zygomycosis (mucormycosis), France, 1997-2006. Emerg. Infect. Dis. 2009; 15:13951401. http://doi.org/10.3201/eid1509.090334

12. Ambrosioni J, Bouchuiguir-wafa K, Garbino J. Emerging invasive zygomycosis in a tertiary care center: Epidemiology and associated risk factors. Int. J. Infect. Dis. 2010; 14:e100-e103. http://doi. org/10.1016/j.ijid.2009.11.024

13. Chakrabarti A, Das A, Mandal J, Shivaprakash MR, George VK, Tarai B, Rao P, Panda N, Verma $\mathrm{SC}$, Sakhuja V. The rising trend of invasive zygomycosis in patients with uncontrolled diabetes mellitus. Med. Mycol. 2006;44:335-342. http://doi. org/10.1080/13693780500464930

14. Skiada A, Pagano L, Groll A et al. Zygomycosis in Europe: Analysis of 230 cases accrued by the registry of the European Confederation of Medical Mycology
(ECMM) Working Group on Zygomycosis between 2005 and 2007. Clin. Microbiol. Infect. 2011; 17:1859-1867. http://doi.org/10.1111/j.14690691.2010.03456.x

15. Rammaert B., Lanternier F., Zahar J. Healthcareassociated mucormycosis. Clin. Infect. Dis. 2012; 54(Suppl. 1):S44-S54. http://doi.org/10.1093/cid/ cir867

16. Rootman J, Robertson W, Lapointe JS. Inflammatory diseases Diseases of the Orbit. A Multidisciplinary Approach.1st ed J. B. Lippincott Company 1988:1556.

17. Brunet K, Rammaert B. Mucormycosis treatment: Recommendations, latest advances, and perspectives J Mycol Med 2020;30:101007. http://doi. org/10.1016/j.mycmed.2020.101007

18. Nithyanandam S, Jacob MS, Battu RR, et al. Rhinoorbito-cerebral mucormycosis. A retrospective analysis of clinical features and treatment outcomes Indian J Ophthalmol. 2003; 51:231-6.

19. Revannavar SM, Supriya PS, Samaga L, Vineeth VK. COVID-19 triggering mucormycosis in a susceptible patient: a new phenomenon in the developing world? BMJ Case Reports CP 2021; 14: e241663. https://doi.org/10.1136/bcr-2021-241663 